\title{
LATE POSTPARTUM PREECLAMPSIA WITH POSTERIOR REVERSIBLE ENCEPHALOPATHY SYNDROME
}

RITESH KAUNTIA, ROHITH VALSALAN, SHUBHA SESHADRI, VINAY R PANDIT, M. M. PRABHU

\section{ABSTRACT}

Posterior reversible encephalopathy syndrome is a reversible syndrome characterized by headache, seizures, altered mentation, and loss of vision associated with white matter changes on imaging. We report here a 27 year-old lady three weeks postpartum, presenting with posterior reversible encephalopathy syndrome. She was treated successfully with antihypertensives and showed dramatic improvement. This condition is important to recognize and needs to be treated promptly to prevent morbidity and mortality in pregnancy and postpartum.

Key words: Posterior reversible leukoencephalopathy syndrome, postpartum, preeclampsia

DOI: $10.4103 / 0019-5359.58880$

\section{INTRODUCTION}

Reversible posterior leukoencephalopathy syndrome (RPLS) or posterior reversible encephalopathy syndrome (PRES) was first described by Hinchey in 1996. ${ }^{[1]}$ She described it as a reversible syndrome manifested as headache, altered mental functioning, seizures, and loss of vision associated with white matter changes, suggestive of edema mainly in the posterior regions of the cerebral hemispheres, but also involving the brainstem, cerebellum, and

Department of Medicine, Kasturba Medical College, Manipal, India

Correspondence:

Rohith Valsalan,

Department of Medicine,

Kasturba Medical College,

Manipal, India.

E-mail: drrohithv@gmail.com other cerebral areas. Postpartum preeclampsia is a rare and under-recognized condition occurring in $5.7 \%$ of all cases of pregnancy-induced hypertension. ${ }^{[2]}$ PRES is a very rare condition and so is usually not suspected. This causes delay in diagnosis and treatment, which can lead to permanent neurological damage. We report here a case of postpartum preeclampsia presenting with PRES, emphasizing the fact that early diagnosis and treatment can prevent complications.

\section{CASE REPORT}

A 27 year-old lady presented to the emergency department three weeks postpartum after she experienced headache, blurring of vision, nausea, and vomiting for one day. She had a normal pregnancy and all her antenatal 
blood pressure recordings were normal. She had undergone a caesarean section in a local hospital due to nonprogression of labor; postoperative and the subsequent puerperal periods were uneventful. Examination revealed that she was conscious, oriented, and afebrile with a blood pressure of 200/110 $\mathrm{mm}$ of $\mathrm{Hg}$. The results of her general examination were unremarkable. A central nervous system examination revealed diminution of vision in both eyes with regard to perception of hand movement. Pupillary reactions and results of a fundoscopic examination were normal and plantars were flexor. Results of abdominal, cardiovascular, and respiratory system examinations were unremarkable. Her complete blood picture, kidney function test, liver function test, clotting parameters, and electrocardiogram were within normal limits. Urine examination revealed $2+$ proteinuria. Echocardiogram did not show any evidence of

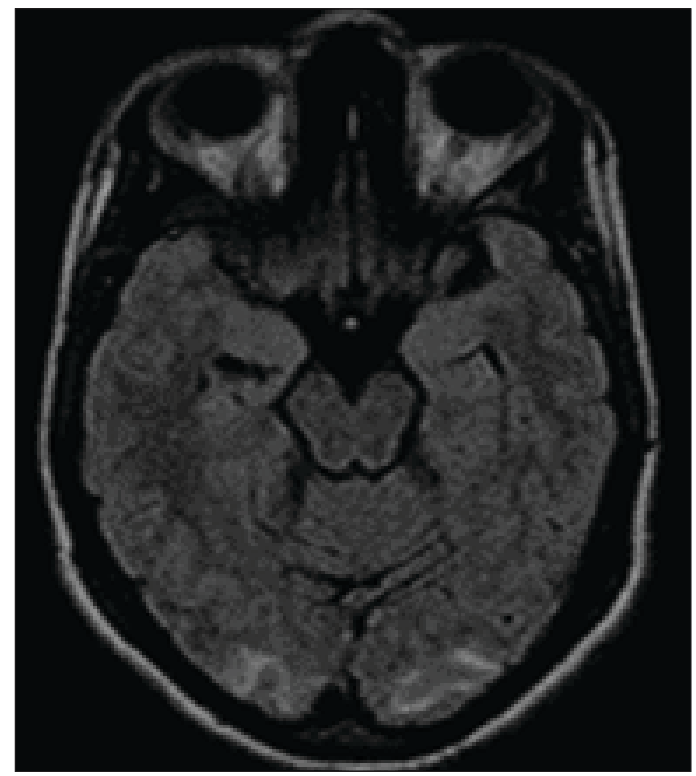

Figure 1: FLAIR sequence of $M R I$ of the brain demonstrating hyperintensity in the white matter of the bilateral occipital lobe left ventricular hypertrophy. MRI of the brain showed symmetrical hyperintense signals in the white matter of the bilateral occipital lobes in T2-weighted and FLAIR sequences [Figure 1]. MRV was done to examine the deep venous system which was found to be normal. She was started on antihypertensives (amlodipine and labetolol), and her vision improved to $6 / 6$ after 48 hours following commencement of the treatment. She was followed up and MRI at one month after initiation of treatment showed disappearance of the hyperintensities [Figure 2].

\section{DISCUSSION}

PRES has become better recognized with the progress made in imaging modalities. Most cases are associated with hypertensive emergencies, allogenic bone marrow transplantation, solid organ transplantation,

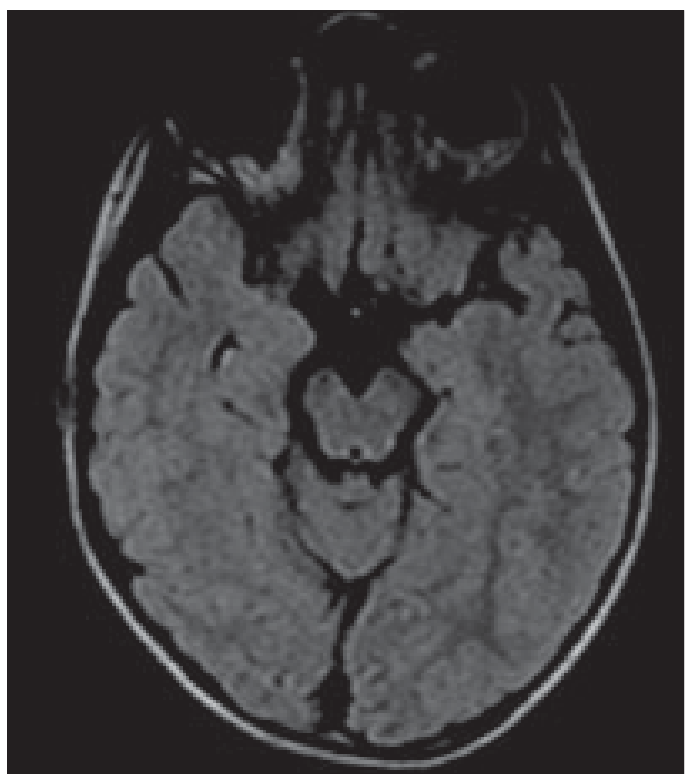

Figure 2: Follow-up MRI of the brain demonstrating disappearance of hyperintensity 
autoimmune disease, high-dose chemotherapy, toxemia of pregnancy, cryoglobulinemia, and thrombotic thrombocytopenic purpura. ${ }^{[1,3]}$ Two theories have been proposed to explain the pathophysiology. The more popular theory suggests that hypertension leads to failure of autoregulation, subsequent hyperperfusion, and vasogenic edema. The other theory suggests that vasoconstriction and hypoperfusion leads to brain ischemia and subsequent vasogenic edema. ${ }^{[4]}$

PRES is characterized by high signal intensity on T2-weighted and FLAIR images, predominantly in the posterior regions, which is caused by subcortical white matter vasogenic edema. Supplemental diffuse weighted imaging (DWI) and apparent diffusion coefficient (ADC) map images are helpful in distinguishing vasogenic from cytotoxic edema, which represents foci of irreversible ischemia. Atypical features have been seen in neuroimaging like significant anterior, cortical, brainstem lesions, recurrent RPLS episodes, foci of permanent injury, hemorrhage into lesions, and unilaterality. ${ }^{[5]}$

Preeclampsia is characterized by hypertension and proteinuria during the antepartum and postpartum periods. When associated with a new onset of seizures which is not attributable to other causes, it is termed eclampsia. Usually it occurs between 20 weeks of gestation and 48 hours postpartum. If it occurs after 48 hours and up to 30 days after delivery, it is called late postpartum preeclampsia or eclampsia., $[2,6,7]$

Our patient presented with features suggestive of preeclampsia with cortical blindness. The differential diagnoses considered were cerebral venous sinus thrombosis, pregnancy-related stroke, reversible cerebral vasoconstriction syndrome (RCVS) with PRES, and postpartum preeclampsia with PRES ${ }^{[8]}$ An MRV (magnetic resonance venogram) was taken to rule out venous sinus thrombosis and its results were normal. As DWI and ADC imaging facilities were not available, vasogenic edema could not be distinguished from cytotoxic edema. Pregnancy-related stroke was an unlikely diagnosis as the patient recovered dramatically and the MRI lesion disappeared on follow-up. Therefore, a diagnosis of PRES was made. RCVS is a differential diagnosis which was considered but we thought PRES is due to failure of autoregulation and vasogenic edema. MR arteriogram could not be done to rule it out.

The difficulty in diagnosis of late postpartum preeclampsia is that there are no preceding symptoms during or after delivery. Most cases of postpartum eclampsia begin abruptly with headache days to weeks following delivery. ${ }^{[9]}$ They also have associated visual disturbances and can progress to eclampsia. Other complications can include aspiration pneumonia, pulmonary edema, disseminated intravascular coagulation, permanent neurological deficit, encephalopathy, and death. PRES is reversible after appropriate treatment, which makes it important to recognize and treat the etiology to prevent its progress to irreversible damage. [10] This case report demonstrates that early treatment with control of blood pressure can reverse this condition and also prevent progression to eclampsia, thus emphasizing the need for early diagnosis and treatment. Thus, patients should be warned of preeclampsia symptoms, not only in the antenatal period but also in the postpartum period so that this condition can be recognized early. ${ }^{[10,11]}$ 


\section{REFERENCES}

1. Hinchey J, Chaves C, Appignani B, Breen J, Pao L, Wang A, et al. A reversible posterior leukoencephalopathy syndrome. N Engl J Med 1996;334:494-500.

2. Matthys LA, Coppage KH, Lambers DS, Barton JR, Sibai BM. Delayed postpartum preeclampsia: An experience of 151 cases. Am J Obstet Gynecol 2004;190:1464-6.

3. Lee VH, Wijdicks EF, Manno EM, Rabinstein AA. Clinical spectrum of reversible posterior leukoencephalopathy syndrome. Arch Neurol 2008;65:205-10.

4. Bartynski WS. Posterior reversible encephalopathy syndrome, part 2: Controversies surrounding pathophysiology of vasogenic edema. AJNR Am J Neuroradiol 2008;29:1043-9.

5. Bartynski WS, Boardman JF. Distinct imaging patterns and lesion distribution in posterior reversible encephalopathy syndrome. AJNR Am J Neuroradiol 2007;28:1320-7.

6. Sibai BM. Diagnosis, prevention, and management of eclampsia. Obstet Gynecol 2005;105:402-10.
7. Lubarsky SL, Barton JR, Friedman SA, Nasreddine S, Ramadan MK, Sibai BM. Late postpartum eclampsia revisited. Obstet Gynecol 1994;83:5025.

8. Singhal AB, Kimberly WT, Schaefer PW, HedleyWhyte ET. Case records of Massachusetts General Hospital: Case 8-2009: A 36-year-old woman with headache, hypertension, and seizure 2 weeks post partum. N Engl J Med 2009;360:1126-37.

9. Schwartz RB, Feske SK, Polak JF, DeGirolami U, laia A, Beckner KM, et al. Preeclampsia-eclampsia: Clinical and neuroradiographic correlates and insight into the pathogenesis of hypertensive encephalopathy. Radiology 2000;217:317-26.

10. Chames MC, Livingston JC, Ivester TS, Barton JR, Sibai BM. Late postpartum eclampsia: A preventable disease? Am J Obstet Gynecol 2002;186:1174-7.

11. Pizon AF, Wolfson AB. Postpartum focal neurologic deficits: Posterior leukoencephalopathy syndrome. J Emerg Med 2005;29:163-6.

Source of Support: Nil. Conflict of Interest: None declared.

\section{Author Help: Reference checking facility}

The manuscript system (www.journalonweb.com) allows the authors to check and verify the accuracy and style of references. The tool checks the references with PubMed as per a predefined style. Authors are encouraged to use this facility, before submitting articles to the journal.

- The style as well as bibliographic elements should be $100 \%$ accurate, to help get the references verified from the system. Even a single spelling error or addition of issue number/month of publication will lead to an error when verifying the reference.

- $\quad$ Example of a correct style Sheahan P, O'leary G, Lee G, Fitzgibbon J. Cystic cervical metastases: Incidence and diagnosis using fine needle aspiration biopsy. Otolaryngol Head Neck Surg 2002;127:294-8.

- Only the references from journals indexed in PubMed will be checked.

- $\quad$ Enter each reference in new line, without a serial number.

- $\quad$ Add up to a maximum of 15 references at a time.

- If the reference is correct for its bibliographic elements and punctuations, it will be shown as CORRECT and a link to the correct article in PubMed will be given.

- If any of the bibliographic elements are missing, incorrect or extra (such as issue number), it will be shown as INCORRECT and link to possible articles in PubMed will be given. 